\title{
Values of Nursing Practice in Caring Type 1 Children
}

\author{
Mrs. Anjuladevi Kamaraj*1, Dr. M. Gandhimathi ${ }^{2}$ and Dr.C.Ramesh ${ }^{3}$ \\ ${ }^{1}$ Research Scholar, Annamalai University, Annamalai Nagar \\ ${ }^{2}$ Professor, Rani Meyyammai College of Nursing, Annamalai University, Annamalai Nagar \\ ${ }^{3}$ Director of Juvenile Diabetes Projects, VHS, Chennai
}

\begin{abstract}
Nursing is a caring profession. Nursing has a high value on the delivery of safe and quality care. Values are a set of personal beliefs and attitudes about the truth, beauty, and worth of any thoughts, object or behavior ${ }^{l .}$ Values serve as guide to life. Values are action oriented and give direction and meaning to one's life. Some of the essential core values for nurses are altruism, autonomy, equality, dignity, integrity, social justice and truth. The caring professional nurse integrates these values in clinical practice. These values represent the responsibility and duty to the public, reflecting expanded nursing roles in the current health care environment ${ }^{2}$. This paper explains the values of nursing practice in caring type 1 diabetes children.
\end{abstract}

Key words: Nursing practice, values, diabetic children, insulin, diet

\section{Introduction}

The nurses need to balance truth, caring, harmony, duty and responsibility. Attitudes, beliefs and behaviours are often linked with values but are not the same as values ${ }^{3}$. An attitude is ones disposition towards an object or a situation. It can be a mental or emotional mind set. It can be positive or negative. Beliefs are ideas that one accepts as truth, they may be expressed by opinions, decisions and creed. Behaviours are observable actions. The values reflect the needs, response to situations, relationship to significant ones, culture, religion and society ${ }^{4}$. A system of values and principles would assist practitioners to respond to complex health issues that have multiple interrelated determinants ${ }^{5}$.

\section{Code of Values in Nursing Practice}

The American Association of colleges of Nursing measures the following as code of ethics in nursing practice ${ }^{6}$ :

- Compassion and respect

- Commitment to client, family and community

- Advocacy for health and safety.

- Responsibility and accountability for individual practice and responsibility to preserve dignity.

- Competence and continued personal and professional growth to ensure the quality of care.

- To collaborate with others and to maintain the integrity of the profession.

In order to maintain the intensive knowledge required for the care of patients, families and communities, lifelong learning is inevitable. Also, the responsibility of individuals is needed to maintain a high level of professional practice and competence through a continuous process of self-evaluation. Nurses have a duty to remain consistent with both their personal and professional values. Individual nurses have a responsibility for the quality of nursing care that their patients receive and are accountable for their own practice. The accountability for individual nursing practice and the appropriate delegation and supervision of tasks should be adhered while providing optimum patient care.

Each individual has a right to ask questions and to make informed choices for their health and future. The nurse's primary commitment is to the patient, whether an individual, family, group or community. The nurse promotes, advocates for, and strives to protect the health, safety, and rights of the patient. Individuals need to work together, using their unique skills and knowledge to deliver better healthcare to the community. Illness with restricted physical ability and being confined to bed can compromise the dignity of patients $^{7}$. Irrespective of the social or economic status, personal attributes, or the nature of health problems, one should work with dignity, respect and compassion. The nurse is responsible for recognizing and maintaining boundaries with colleagues and patients. Nurses actively participate in assessing and advocating for the responsible and appropriate use of interventions to minimize unwanted treatment and patient suffering.

\section{Brian Hall Model (1991) of Values and its Application on Diabetic Children}

Brain Hall ${ }^{8}$ identified 3 levels of valuing viz. Foundation, focus and future. In this model, two kinds of behavior indicate a held value. One kind is habitual; the person does not have to think about it. For example, diabetic child adopts monitoring of blood and urine glucose, taking regular insulin, recommended diet and following regular exercise. Maintaining these habits would indicate a foundation value. Another kind of 
behavior lies on choice. ie. the person does this behavior only when he or she sets it as a goal. For example, Nurse advises the child to check his or her urine glucose 4 times a day. To add this procedure as routine, the child would need to purchase diatrix and allot additional time for using it. He or she might also need to set the alarm to get up earlier. Until these additional behaviours become habits, the extra diabetic care would remain a choice or focus value. On the other hand, the nurse has to teach about buying diatrix, explain how to use it and its health benefits. When the diabetic child adheres to the instructions given by the diabetologist, dietitian and the nurse, the early and late complications can be prevented. The diabetic child requires depth knowledge to manage his or her daily life successfully. Then the value of additional diabetic care would remain a vision or future value. It would not become real until a diabetic child acted on it. The diabetic child ultimately would like to hold the future values. But they often lack the knowledge or skills necessary to integrate into their lives.

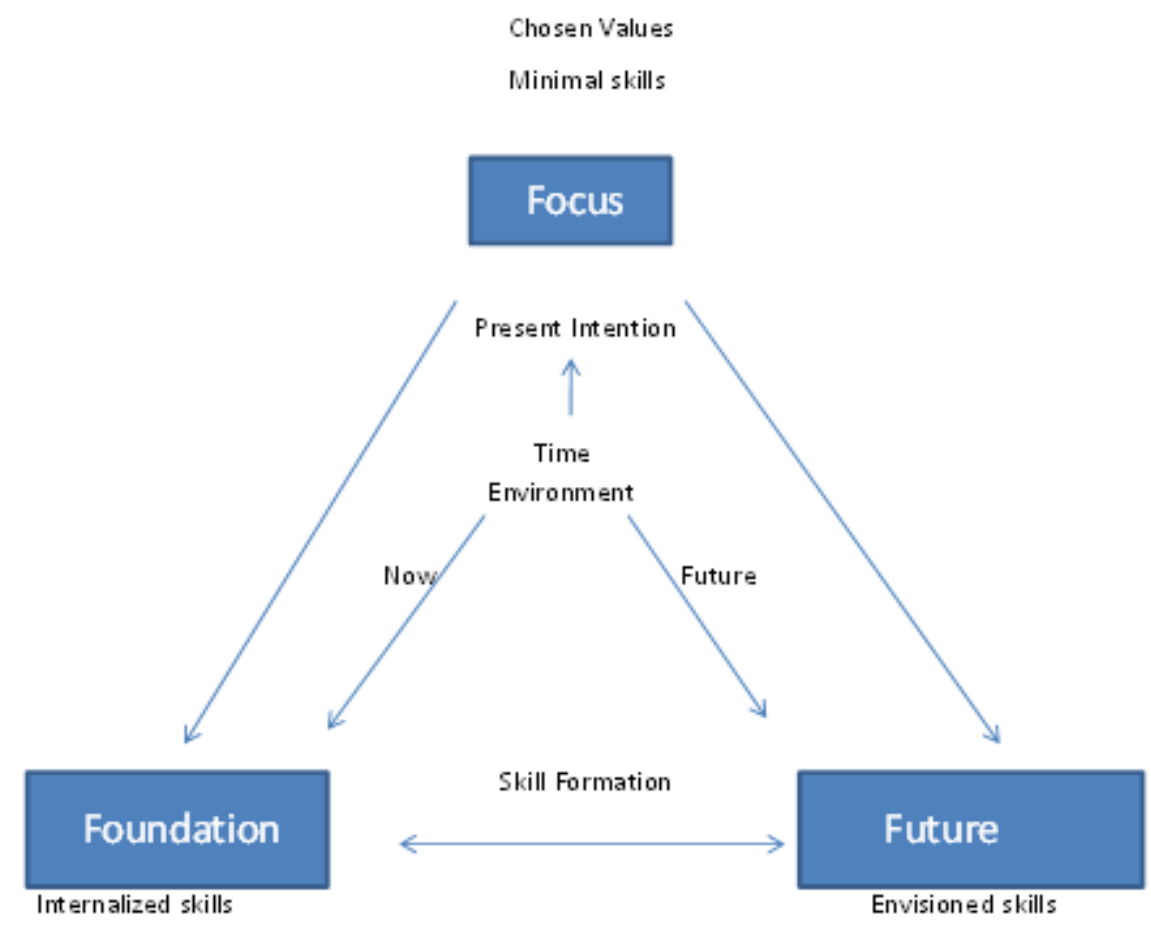

Fig. 1. Brian Hall model of values (1991)

The role of nurses is to promote a positive environment for health behavioral change. In combination with therapeutic, motivational and communication techniques, nurses can assist clients to progress in accepting more healthful behavior? ${ }^{9}$.

\section{Values of Nursing Practice in Caring Type 1 Children}

The diagnosis of a chronic disease that requires daily management can be difficult to accept. Support for the child depends on his or her age and developmental stage. The child should be encouraged to express the feelings about the disease and its management. The child's developmental stage and cognitive level influence his or her readiness to take on responsibility for self care. For example, school age children can learn to test urine/blood glucose, administer insulin and keep records.

The parents particularly talk with the adolescent to assess his or her mood and evaluate their motivation to manage the meal plan, exercise regimen, blood glucose monitoring and insulin therapy. The parents should associate with the adolescent in order to carry out the assumed care independently under their supervision. Type 1 diabetes focuses on glycemic control by combining insulin, nutrition management to support growth and maintain blood glucose at near normal level, an exercise regimen and psycho-social support. The nurse is an important member of the management team and she is responsible for educating the child and family. The initial teaching focuses on the survival skills necessary for home management which includes insulin administration, blood/urine glucose testing, record keeping, meal planning and recognition and treatment of hypo and hyper glycemia. The goal of insulin therapy is to maintain a range of blood glucose levels that vary by the Child's age. Insulin therapy is evaluated every three months with a $\mathrm{HbA} 1_{c}$ level. Insulin requirement does not remain constant but change continuously during growth and development and the needs vary according to child's activity level and puberty status. In the event of poor glycemic control, vascular changes can appear as early as 2.5 - 3 years after diagnosis. High blood glucose levels may impair the ability of granulocytes to function 
normally and can depress leukocyte phagocytosis. All infections cause an increased need for insulin. However, with good to excellent glycemic control, vascular changes could be postponed to 10 years or more. The complexity of the disease and its management requires that the child and family incorporate diabetes needs into their life style. The insulin should never be omitted during an illness. Urinary ketones should be checked during nausea, vomiting, diarrhea, fever and illness.

The child must have an adequate calorie intake to prevent hypoglycemia. Daily calorie requirements are individualized for each child according his or her needs to support growth and development for disease management. Generally, one unit of insulin covers $8 \mathrm{gm}$ of carbohydrates making insulin dosage calculation for meal coverage relatively easy. A high fibre diet is also recommended for improved control of blood glucose. Eating at consistent interval is important for glycemic control (3 meals and 3 snacks a day). The child and parents need to learn about the relationship between foods consumed and insulin needed. Meal plan also needs to be adjusted for exercise. Food may become a battle ground for toddlers who are picky eaters, but must eat enough for the insulin dosage. The distribution of calories should be calculated to fit the activity pattern of each child. Reinforce the importance of balancing dietary intake, insulin and exercise every day. Evaluation for the potential complications of diabetes should be performed annually including blood for lipid levels, blood pressure, renal function, urine for albumin and ophthalmologic examination for retinopathy . All children with diabetes should carry sugar containing candy (5 nos) or sugar cubes (10-15 gm) of simple carbohydrates and alleviate the symptoms of hypoglycemia. This is given in 15-20 min. when the child is conscious. If a child does not respond to the treatment, the child shoud be transported to a hospital.

The child with diabetes should be treated as any other child without a chronic condition, including a limit setting and consistent discipline for unacceptable behavior. The children with type 1 diabetes may learn mal adaptive behaviours using their disease to obtain something they want. The parents should be taught to be alert to signs of maladaptive such as helplessness, demanding or whining behaviours and any evidence of poor coping. Additional behaviours may include skipping blood glucose testing and damaging / loosing equipment. Most children are able to keep the disease under control with periodic assessment and adjusting of insulin, diet and activity under the supervision of the practioners. Education is a long term nursing activity as family and patient needs change and new findings are applied. Family relationships play an important role in diabetes management. Studies have shown that low levels of conflict, high levels of cohesion, organization and good communication patterns are associated with better regimen adherence. Social support also serves to buffer the adverse effect of stress on diabetic management.

When the nurse has a specialized knowledge on diabetic management, she has a confidence, hope and openness which ensure freedom to take suitable decision when ever required. The appropriateness of nursing care can be evaluated by comparing the care planned and the care delivered to patients to the standards of care ${ }^{10}$.

\section{Conclusion}

The values are not stable but vary among individuals and change according to life experiences and the level of maturity. The values have an impact on most of one's decisions. Nurses need to understand their own values and be sensitive to others to provide health care effectively.

\section{Acknowledgement}

One of the authors Mrs. Anjuladevi Kamaraj thanks Dr.Vijayalakshmi, Principal, Rani Meyyammai College of Nursing and Dr. C.V. Krishnaswami, Professor and Head, Juvenile Diabetes Research Center, VHS for their encouragement and support.

\section{References}

[1] Dorothy R Marlow and Barbera A Redding, Text book of pediatric nursing, 1998, W B Saunders Co., Philadelphia.

[2] Hook, K, 2001, Empowered caring and the code of ethics, The American Nurses Association Ethics and Human rights issues update.

[3] Hall B, 1982, The personal discernment inventory, New York, Paulist Press.

[4] Ruth F Craven and Constance J Hirnle, Fundamentals of nursing, $6^{\text {th }}$ Edition, 2009, Wolters Kluwea, Philadelphia.

[5] Jane Gregg and Lily O'Hara, Values and Principles evident in current health promotion practice, Health Promotion Journal of Australia: 2007; 18(1)

[6] American Association of Colleges of Nursing,1998, Essentials of baccalaureate education for professional nursing practice, Washington D.C.

[7] Nahid Dehghan Nayeri et. al.,Iranian nurses and hospitalized teen agers' views of dignity, Nurs Ethics, 2011,18:474-84.

[8] Hall B, 1991, Spiritual connections; the journey of discipleship and Christian values, Dayton, OH; values technology inc.

[9] Donna L Wong et. al. Whaley and Wong's Nursing care of infants and children, 2008, Mosby publication, New York

[10] 10.Diane Irvine et.al.,Finding value in nursing care:A frame work for quality improvement and clinical evaluation, Health and Nursing economics, May- June, 1998. 